\title{
Null Curves and Representation in Three Dimensional Minkowski Spacetime
}

\author{
Huili Liu ${ }^{1 *}$ and Seoung Dal Jung ${ }^{2}$ \\ ${ }^{1}$ Department of Mathematics, Northeastern University, China \\ Email: liuhl@mail.neu.edu.cn \\ ${ }^{2}$ Department of Mathematics and Research Institute for Basic Sciences, Jeju National University, Korea \\ Email: sdjung@jejunu.ac.kr
}

\begin{abstract}
In this paper we give a kind of representation formula for a null curve with null arc length parameter in three dimensional Minkowski spacetime $\mathbb{E}_{1}^{3}$ and define structure function of such null curve. Using this representation formula and relations between the null curvature function and structure function of null curve, especially by solving certain differential equations, we obtain some characterizations and classifications of some special null curves.
\end{abstract}

Mathematics Subject Classification (2000): 53A35, 53B30, 53B50.

Keywords: Minkowski spacetime; null curve; structure function; curvature function; torsion function; special curve.

\section{Introduction}

The curves theory of Minkowski spacetime (or called Minkowski space) is very important and interesting in both physics and mathematics. Locally, there exist spacelike curves, timelike curves and null curves (lightlike curves) in Minkowski space. For the study of the curves in Minkowski space, there are many more differences between the null curves and the spacelike curves or timelike curves. A. Nersessian etc. studied geometrical particle model associated with null paths (null curves) in Minkowski spacetime ([11]-[14]). W. Bonnor, G. Clément, A. Ferrández etc. studied the null curves in Minkowski space ([1], [3]-[8]). In this paper, we consider the null curves in three dimensional Minkowski spacetime (simply, Minkowski 3 -space). At first we deal with the null arc length parameter and the relation with the arbitrary parameter of the null curve. Then we give a kind of representation formula for the null curve with the null arc length parameter in Minkowski 3-space $\mathbb{E}_{1}^{3}$ and define structure function of such null curve. Using this representation formula and the characters of the structure function of the null curve, by solving certain differential equations, we characterize some special null curves and obtain certain classification results.

This paper is arranged as follows. In Section 2 we discuss the arc length parameter of the null curve and define the null curvature function and null Frenet frames of the null curve. Then we give the relation of the null arc length parameter and the arbitrary parameter of the null curve. In section 3, using the tangent indicatrix of the null curve we give a kind of representation formula for the null curve with the null arc length parameter in Minkowski 3-space and define the structure function of the null curve. Then the relation between the null curvature function and the structure function is given. In section 4 we discuss some special null curves, for examples, helix, rectifying curves etc. and obtain some classification results.

\section{$2 \quad$ Null Curves in Minkowski 3-space $\mathbb{E}_{1}^{3}$}

Let $r(t)$ be a null curve in Minkowski 3-space $\mathbb{E}_{1}^{3}$ with the indefinite Minkowski inner product

$$
\langle a, b\rangle=a_{1} b_{1}+a_{2} b_{2}-a_{3} b_{3},
$$

for $a=\left(a_{1}, a_{2}, a_{3}\right)$ and $b=\left(b_{1}, b_{2}, b_{3}\right) \in \mathbb{E}_{1}^{3}$. Putting $\frac{\mathrm{d} r(t)}{\mathrm{d} t}=r^{\prime}(t)$, since $\left\langle r^{\prime}(t), r^{\prime}(t)\right\rangle=0$, we have $\left\langle r^{\prime \prime}(t), r^{\prime}(t)\right\rangle=0$. In the following we always assume that $r^{\prime \prime}(t) \nVdash r^{\prime}(t)$. In this case, $r^{\prime \prime}(t)$ is spacelike, so 
we can choose the parameter $t$ such that $\left\langle r^{\prime \prime}(t), r^{\prime \prime}(t)\right\rangle=1$. By the parameter transformation $s=s(t)$, putting $\frac{\mathrm{d} r(s)}{\mathrm{d} s}=\dot{r}(s)$, we have

$$
\left\{\begin{array}{l}
\dot{r}(s)=r^{\prime}(t) \frac{\mathrm{d} t}{\mathrm{~d} s} \\
\ddot{r}(s)=r^{\prime \prime}(t)\left(\frac{\mathrm{d} t}{\mathrm{~d} s}\right)^{2}+r^{\prime}(t) \frac{\mathrm{d}^{2} t}{\mathrm{~d} s^{2}} \\
\dddot{r}(s)=r^{\prime \prime \prime}(t)\left(\frac{\mathrm{d} t}{\mathrm{~d} s}\right)^{3}+3 r^{\prime \prime}(t)\left(\frac{\mathrm{d} t}{\mathrm{~d} s}\right)\left(\frac{\mathrm{d}^{2} t}{\mathrm{~d} s^{2}}\right)+r^{\prime}(t) \frac{\mathrm{d}^{3} t}{\mathrm{~d} s^{3}} .
\end{array}\right.
$$

Then

$$
\langle\ddot{r}(s), \ddot{r}(s)\rangle=\left\langle r^{\prime \prime}(t), r^{\prime \prime}(t)\right\rangle\left(\frac{\mathrm{d} t}{\mathrm{~d} s}\right)^{4}
$$

Hence

$$
\sqrt[4]{\langle\ddot{r}(s), \ddot{r}(s)\rangle} \mathrm{d} s= \pm \sqrt[4]{\left\langle r^{\prime \prime}(t), r^{\prime \prime}(t)\right\rangle} \mathrm{d} t
$$

yields that

$$
\sqrt[4]{\left\langle r^{\prime \prime}(t), r^{\prime \prime}(t)\right\rangle} \mathrm{d} t
$$

is an invariant of the null curve $r(t)$ in Minkowski 3-space $\mathbb{E}_{1}^{3}$. Therefore the parameter $s$ of the null curve $r(s)$ is uniquely defined such that $\langle\ddot{r}(s), \ddot{r}(s)\rangle=1$ in the sense of the parameter change $s \rightarrow-s$.

Definition 2.1. Let $r(s)$ be a null curve in Minkowski 3-space $\mathbb{E}_{1}^{3}$ with parameter s. If $\langle\ddot{r}(s), \ddot{r}(s)\rangle \equiv 1$ we call s null arc length parameter (simply, arc length parameter) of the null curve $r(s)$ in $\mathbb{E}_{1}^{3}$.

Let $r(s)$ be a null curve in Minkowski 3-space $\mathbb{E}_{1}^{3}$ with the arc length parameter $s$. Putting $\dot{r}(s)=x(s)$, $\ddot{r}(s)=\dot{x}(s)=\alpha(s)$ and

$$
y(s)=-\ddot{x}(s)-\frac{1}{2}\langle\ddot{x}(s), \ddot{x}(s)\rangle x(s)=-\dddot{r}(s)-\frac{1}{2}\langle\dddot{r}(s), \dddot{r}(s)\rangle \dot{r}(s),
$$

we have

$$
\left\{\begin{aligned}
\langle x(s), x(s)\rangle=\langle y(s), y(s)\rangle= & \langle x(s), \alpha(s)\rangle=\langle\alpha(s), y(s)\rangle=0, \\
& \langle x(s), y(s)\rangle=\langle\alpha(s), \alpha(s)\rangle=1 .
\end{aligned}\right.
$$

Therefore we know that $\{x(s), \alpha(s), y(s)\}$ forms an asymptotic orthonormal frame along the null curve $r(s)$ and the Frenet formulas of $r(s)$ are given by

$$
\left\{\begin{array}{l}
\dot{r}(s)=x(s), \\
\dot{x}(s)=\alpha(s), \\
\dot{\alpha}(s)=\kappa(s) x(s)-y(s), \\
\dot{y}(s)=-\kappa(s) \alpha(s) .
\end{array}\right.
$$

Definition 2.2. The function $\kappa(s)$ defined in (8) is called standard null curvature function or null curvature function (simply, curvature) of the null curve $r(s)$ with the null arc length parameter $s$ in $\mathbb{E}_{1}^{3}$. The frame $\{x(s), \alpha(s), y(s)\}$ is called null Frenet frame of the null curve $r(s)$.

From (8) we have

$$
\kappa(s)=-\frac{1}{2}\langle\dddot{r}(s), \dddot{r}(s)\rangle,
$$

and

$$
\dddot{r}(s)-2 \kappa(s) \ddot{r}(s)-\dot{\kappa}(s) \dot{r}(s)=0 .
$$

Then by $(2),\langle\ddot{r}(s), \ddot{r}(s)\rangle=1$ and (6), for the arbitrary parameter $t$ of the null curve $r(t)$, we get

$$
\left\{\begin{array}{l}
\left(\frac{\mathrm{d} t}{\mathrm{~d} s}\right)=\left\langle r^{\prime \prime}, r^{\prime \prime}\right\rangle^{-\frac{1}{4}}, \\
\left(\frac{\mathrm{d}^{2} t}{\mathrm{~d} s^{2}}\right)=-\frac{1}{2}\left\langle r^{\prime \prime}, r^{\prime \prime}\right\rangle^{-\frac{3}{2}}\left\langle r^{\prime \prime}, r^{\prime \prime \prime}\right\rangle, \\
\left(\frac{\mathrm{d}^{3} t}{\mathrm{~d} s^{3}}\right)=\frac{3}{2}\left\langle r^{\prime \prime}, r^{\prime \prime}\right\rangle^{-\frac{11}{4}}\left\langle r^{\prime \prime}, r^{\prime \prime \prime}\right\rangle^{2}-\frac{1}{2}\left\langle r^{\prime \prime}, r^{\prime \prime}\right\rangle^{-\frac{7}{4}}\left[\left\langle r^{\prime \prime \prime}, r^{\prime \prime \prime}\right\rangle+\left\langle r^{\prime \prime}, r^{\prime \prime \prime \prime}\right\rangle\right],
\end{array}\right.
$$


and

$$
\begin{aligned}
-2 \kappa(t)= & \left\langle r^{\prime \prime \prime}, r^{\prime \prime \prime}\right\rangle\left\langle r^{\prime \prime}, r^{\prime \prime}\right\rangle^{-\frac{3}{2}}+\frac{9}{4}\left\langle r^{\prime \prime}, r^{\prime \prime}\right\rangle^{-\frac{5}{2}}\left\langle r^{\prime \prime}, r^{\prime \prime \prime}\right\rangle^{2} \\
& -3\left\langle r^{\prime \prime}, r^{\prime \prime}\right\rangle^{-\frac{5}{2}}\left\langle r^{\prime \prime}, r^{\prime \prime \prime}\right\rangle^{2}+\left\langle r^{\prime \prime}, r^{\prime \prime}\right\rangle^{-\frac{3}{2}}\left[\left\langle r^{\prime \prime \prime}, r^{\prime \prime \prime}\right\rangle+\left\langle r^{\prime \prime}, r^{\prime \prime \prime \prime}\right\rangle\right] \\
& -3\left\langle r^{\prime \prime}, r^{\prime \prime}\right\rangle^{-\frac{5}{2}}\left\langle r^{\prime \prime}, r^{\prime \prime \prime}\right\rangle^{2},
\end{aligned}
$$

that is

$$
-2 \kappa(t)=2\left\langle r^{\prime \prime}, r^{\prime \prime}\right\rangle^{-\frac{3}{2}}\left\langle r^{\prime \prime \prime}, r^{\prime \prime \prime}\right\rangle-\frac{15}{4}\left\langle r^{\prime \prime}, r^{\prime \prime}\right\rangle^{-\frac{5}{2}}\left\langle r^{\prime \prime}, r^{\prime \prime \prime}\right\rangle^{2}+\left\langle r^{\prime \prime}, r^{\prime \prime}\right\rangle^{-\frac{3}{2}}\left\langle r^{\prime \prime}, r^{\prime \prime \prime \prime}\right\rangle .
$$

Proposition 2.1. Let $r(t)$ be a null curve in Minkowski 3-space $\mathbb{E}_{1}^{3}$ with arbitrary parameter $t$. The null curvature function $\kappa(t)$ of $r(t)$ is given by

$$
\kappa(t)=\frac{-8\left\langle r^{\prime \prime}, r^{\prime \prime}\right\rangle\left\langle r^{\prime \prime \prime}, r^{\prime \prime \prime}\right\rangle+15\left\langle r^{\prime \prime}, r^{\prime \prime \prime}\right\rangle^{2}-4\left\langle r^{\prime \prime}, r^{\prime \prime}\right\rangle\left\langle r^{\prime \prime}, r^{\prime \prime \prime \prime}\right\rangle}{8\left\langle r^{\prime \prime}, r^{\prime \prime}\right\rangle^{\frac{5}{2}}} .
$$

\section{Representation Formulas of the Null Curves}

Let $r(s): \mathbf{I} \rightarrow \mathbb{E}_{1}^{3}$ be a null curve in Minkowski 3-space $\mathbb{E}_{1}^{3}$ with the arc length parameter $s$. Since $\dot{r}(s)$ is lightlike, we know that $\langle\dot{r}(s), \dot{r}(s)\rangle \equiv 0$. Then putting $\dot{r}(s)=\left(\xi_{1}(s), \xi_{2}(s), \xi_{3}(s)\right)$ we have

$$
\xi_{1}^{2}+\xi_{2}^{2}-\xi_{3}^{2}=0 .
$$

From $\xi_{1}^{2}-\xi_{3}^{2}=-\xi_{2}^{2}$ we get

$$
\frac{\xi_{1}+\xi_{3}}{\xi_{2}}=\frac{-\xi_{2}}{\xi_{1}-\xi_{3}}, \quad \text { or } \quad \frac{\xi_{1}+\xi_{3}}{-\xi_{2}}=\frac{\xi_{2}}{\xi_{1}-\xi_{3}} .
$$

Without loss of generality, for a null curve $x: \mathbf{I} \rightarrow \mathbb{E}_{1}^{3}$ with $\dot{r}(s)=x(s)=\left(\xi_{1}(s), \xi_{2}(s), \xi_{3}(s)\right)$, we may assume that

$$
\frac{\xi_{1}+\xi_{3}}{\xi_{2}}=\frac{-\xi_{2}}{\xi_{1}-\xi_{3}}=f(s)
$$

and

$$
\xi_{2}=2 \rho(s)
$$

From (14) and (15) we get

$$
\left\{\begin{aligned}
\xi_{1}+\xi_{3} & =2 \rho f \\
\xi_{1}-\xi_{3} & =-2 \rho f^{-1} \\
\xi_{2} & =2 \rho,
\end{aligned}\right.
$$

therefore,

$$
\left\{\begin{array}{l}
\xi_{1}=\rho\left(f-f^{-1}\right), \\
\xi_{2}=2 \rho \\
\xi_{3}=\rho\left(f+f^{-1}\right) .
\end{array}\right.
$$

That is, the null curve $r(s): \mathbf{I} \rightarrow \mathbb{E}_{1}^{3}$ can be written as

$$
r(s)=\int \dot{r}(s) \mathrm{d} s=\int x(s) \mathrm{d} s=\int\left(\xi_{1}(s), \xi_{2}(s), \xi_{3}(s)\right) \mathrm{d} s=\int \rho\left(f-f^{-1}, 2, f+f^{-1}\right) \mathrm{d} s .
$$

In the following, for the convenience we use

$$
f^{\prime}(s)=\dot{f}(s)=f_{s}(s)=\frac{\mathrm{d} f(s)}{\mathrm{d} s} .
$$


From (18) we have

$$
\ddot{r}(s)=\dot{x}(s)=\rho_{s}\left(f-f^{-1}, 2, f+f^{-1}\right)+\rho f_{s}\left(1+f^{-2}, 0,1-f^{-2}\right) .
$$

Since $s$ is the null arc length parameter of the null curve $r(s)$, we have

$$
4 \rho^{2} f_{s}^{2} f^{-2}=1
$$

Therefore we know that $f_{s} \neq 0$ and by an appropriate transformation, if necessary, we may assume that

$$
\rho(s)=\frac{f(s)}{2 f_{s}(s)} .
$$

Then we get the following conclusion.

Theorem 3.1. Let $r(s): \mathbf{I} \rightarrow \mathbb{E}_{1}^{3}$ be a null curve in $\mathbb{E}_{1}^{3}$ with the null arc length parameter s. Then $r(s)=\left(x_{1}(s), x_{2}(s), x_{3}(s)\right)$ can be written as

$$
\begin{aligned}
r(s) & =\int \frac{f}{2 f_{s}}\left(f-f^{-1}, 2, f+f^{-1}\right) \mathrm{d} s \\
& =\int \frac{1}{2} f_{s}^{-1}\left(f^{2}-1,2 f, f^{2}+1\right) \mathrm{d} s
\end{aligned}
$$

or

$$
\left\{\begin{array}{l}
x_{1}(s)=\int\left(\frac{f^{2}(s)-1}{2 f_{s}(s)}\right) \mathrm{d} s \\
x_{2}(s)=\int\left(\frac{f(s)}{f_{s}(s)}\right) \mathrm{d} s, \\
x_{3}(s)=\int\left(\frac{f^{2}(s)+1}{2 f_{s}(s)}\right) \mathrm{d} s,
\end{array}\right.
$$

for some non constant function $f(s)$. Here, $f_{s}=\frac{\mathrm{d} f}{\mathrm{~d} s}$.

Definition 3.1. The function $f(s)$ in Theorem 3.1 is called structure function of the null curve $r(s)$ : $\mathbf{I} \rightarrow \mathbb{E}_{1}^{3}$ with the null arc length parameter $s$.

In the following, we discuss the relations between the structure function and null curvature function of the null curve. From (21) we have

$$
\begin{aligned}
2 \ddot{r}(s)=2 \dot{x}(s) & =-f_{s}^{-2} f_{s s}\left(f^{2}-1,2 f, f^{2}+1\right)+2(f, 1, f), \\
2 \dddot{r}(s)=2 \ddot{x}(s)= & \left(2 f_{s}^{-3} f_{s s}^{2}-f_{s}^{-2} f_{s s s}\right)\left(f^{2}-1,2 f, f^{2}+1\right) \\
& -2 f_{s}^{-1} f_{s s}(f, 1, f)+2 f_{s}(1,0,1) .
\end{aligned}
$$

Then

$$
\begin{aligned}
\langle\dddot{r}(s), \dddot{r}(s)\rangle=\langle\ddot{x}, \ddot{x}\rangle & =f_{s}^{-2} f_{s s}^{2}-2 f_{s}\left(2 f_{s}^{-3} f_{s s}^{2}-f_{s}^{-2} f_{s s s}\right) \\
& =-\left[\left(\log f_{s}\right)_{s}\right]^{2}+2\left[\left(\log f_{s}\right)_{s}\right]_{s} .
\end{aligned}
$$

From (6) we have

$$
\begin{aligned}
y(s) & =-\dddot{r}(s)-\frac{1}{2}\langle\dddot{r}(s), \dddot{r}(s)\rangle \dot{r}(s) \\
& =-\frac{1}{2} f_{s}^{-2} f_{s s}^{2} x+f_{s}^{-1} f_{s s}(f, 1, f)-f_{s}(1,0,1) .
\end{aligned}
$$

Definition 3.2. Let $r(s): \mathbf{I} \rightarrow \mathbb{E}_{1}^{3}$ be a null curve in $\mathbb{E}_{1}^{3}$ with the null arc length parameter $s$. Then

$$
\bar{r}(s)=\int y(s) \mathrm{d} s
$$

where $y(s)$ is defined by (6), and is also a null curve in $\mathbb{E}_{1}^{3}$ and is called associated null curve (or dual null curve) of the null curve $r(s)$. 
From (9) and (24) we know that the null curvature function $\kappa(s)$ of the null curve $r(s)$ satisfies

$$
\begin{aligned}
\kappa(s) & =-\frac{1}{2}\langle\dddot{r}(s), \dddot{r}(s)\rangle \\
& =\frac{1}{2}\left[\left(\log f_{s}\right)_{s}\right]^{2}-\left[\left(\log f_{s}\right)_{s}\right]_{s} .
\end{aligned}
$$

This is a Riccati equation of $\xi(s)=\left(\log f_{s}\right)_{s}$ and the linear term of it is zero.

Remark 3.1. For the Riccati equation

$$
\xi^{\prime}(s)=\frac{\mathrm{d} \xi(s)}{\mathrm{d} s}=\frac{1}{2} \xi^{2}(s)-\kappa(s)
$$

putting

$$
\eta(s)=\exp \left(-\int \frac{1}{2} \xi(s) \mathrm{d} s\right)
$$

the equation (28) can be written as

$$
\eta^{\prime \prime}(s)-\frac{1}{2} \kappa(s) \eta(s)=0 .
$$

This is a linear equation of second order for $\eta(s)$.

Remark 3.2. Using Theorem 3.1 and the relation (27) we can study the properties of the null curves and construct certain null curves with some characters.

\section{Some Special Null Curves in $\mathbb{E}_{1}^{3}$}

In this section we consider some special null curves in Minkowski 3-space. The null curvature functions of such null curves also give the solutions of the Riccati equation (27) (or liner equation of second order $(30))$.

Theorem 4.1. Let $r(s): \mathbf{I} \rightarrow \mathbb{E}_{1}^{3}$ be a null curve with the null curvature function $\kappa(s)=$ constant and the null arc length parameter $s$. Then $r(s)$ can be written as the following

(i) $r(s)=C_{1} s^{3}+C_{2} s^{2}+C_{3} s$, for $\kappa=0$;

(ii) $r(s)=C_{1} \sinh (\sqrt{2 \kappa}) s+C_{2} \cosh (\sqrt{2 \kappa}) s+C_{3} s$, for $\kappa>0$;

(iii) $r(s)=C_{1} \sin (\sqrt{-2 \kappa}) s+C_{2} \cos (\sqrt{-2 \kappa}) s+C_{3} s$, for $\kappa<0$;

where $C_{1}, C_{2}, C_{3} \in \mathbb{E}_{1}^{3}$.

Proof. From (8) and the null curvature function $\kappa=$ constant, we have

$$
\dddot{r}(s)=2 \kappa \ddot{r}(s) .
$$

Solving this equation we obtain that

$$
\begin{aligned}
& r(s)=C_{1} s^{3}+C_{2} s^{2}+C_{3} s+C_{4}, \quad \kappa=0, \\
& r(s)=C_{1} \sinh (\sqrt{2 \kappa}) s+C_{2} \cosh (\sqrt{2 \kappa}) s+C_{3} s+C_{4}, \quad \kappa>0, \\
& r(s)=C_{1} \sin (\sqrt{-2 \kappa}) s+C_{2} \cos (\sqrt{-2 \kappa}) s+C_{3} s+C_{4}, \quad \kappa<0,
\end{aligned}
$$

where $C_{1}, C_{2}, C_{3}, C_{4} \in \mathbb{E}_{1}^{3}$. By a translation in $\mathbb{E}_{1}^{3}$ we get the conclusion of this theorem.

Corollary 4.2. Let $r(s): \mathbf{I} \rightarrow \mathbb{E}_{1}^{3}$ be a null curve in $\mathbb{E}_{1}^{3}$ with the null arc length parameter $s$ and structure function $f(s)$. If the null curvature function $\kappa(s)$ of $r(s)$ is constant, the structure function $f(s)$ of $r(s)$ satisfies

$$
\left[\left(\log f_{s}\right)_{s}\right]^{2}-2\left(\log f_{s}\right)_{s s}=2 \kappa(s)=c=\text { constant }
$$

and can be written as 
1. when $-a^{2}=c<0, f(s)=\frac{2}{a} \tan \frac{a s}{2}$;

2. when $c=0, f(s)=\frac{-a}{s}$;

3. when $a^{2}=c>0, f(s)=\frac{2}{a} \tanh \frac{a s}{2}$.

Proof. Solving the equation (31) we can get the conclusion ([10]).

Theorem 4.3. Let $r(s): \mathbf{I} \rightarrow \mathbb{E}_{1}^{3}$ be a null curve with the null arc length parameter $s$, null curvature function $\kappa(s)$ and null Frenet frame $\{x(s), \alpha(s), y(s)\}$. If there exists some constant vector $0 \neq C_{0} \in \mathbb{E}_{1}^{3}$ such that $\left\langle\alpha(s), C_{0}\right\rangle$ is constant, $r(s)$ is called a slop null helix and can be written as the curves given in Theorem 4.1 or the following

(i) $r(s)=C_{1} s^{2}+C_{2} s^{(2+\sqrt{1+2 a})}+C_{3} s^{(2-\sqrt{1+2 a})}$, for $2 a>-1$;

(ii) $r(s)=C_{1} s^{2}+C_{2} s^{2} \log s+C_{3} s^{2} \log ^{2} s$, for $2 a=-1$;

(iii) $r(s)=C_{1} s^{2}+C_{2} s^{2} \sin [(\sqrt{-1-2 a}) \log s]+C_{3} s^{2} \cos [(\sqrt{-1-2 a}) \log s]$, for $2 a<-1$;

where $C_{1}, C_{2}, C_{3} \in \mathbb{E}_{1}^{3}$.

Remark 4.1. In [9], situation like case (iii) is inadvertently missed out.

Proof. From $(8)$ and $\left\langle\alpha(s), C_{0}\right\rangle=$ constant, by a calculation we know that the null curvature function $\kappa(s)$ is constant or satisfies

$$
2\left(\frac{\kappa^{\prime}}{\kappa}\right)^{\prime}-\left(\frac{\kappa^{\prime}}{\kappa}\right)^{2}=0,
$$

that is

$$
2 \kappa \kappa^{\prime \prime}-3\left(\kappa^{\prime}\right)^{2}=0
$$

The solution of $(32)$ is

$$
\kappa(s)=a(s+b)^{-2},
$$

where $a$ and $b$ are integral constants. By a parameter transformation we may put $b=0$. From (10) we get

$$
s^{3} \dddot{x}(s)-2 a s \dot{x}(s)+2 a x(s)=0 .
$$

The solutions of the Euler equation (34) are

(i) $x(s)=B_{1} s+B_{2} s^{(1+\sqrt{1+2 a})}+B_{3} s^{(1-\sqrt{1+2 a})}$, for $2 a>-1$;

(ii) $x(s)=B_{1} s+B_{2} s \log s+B_{3} s \log ^{2} s$, for $2 a=-1$;

(iii) $x(s)=B_{1} s+B_{2} s \sin [(\sqrt{-1-2 a}) \log s]+B_{3} s \cos [(\sqrt{-1-2 a}) \log s]$, for $2 a<-1$;

where $B_{1}, B_{2}, B_{3} \in \mathbb{E}_{1}^{3}$. With $\dot{r}(s)=x(s)$ we get the conclusion of this theorem.

Corollary 4.4. Let $r(s): \mathbf{I} \rightarrow \mathbb{E}_{1}^{3}$ be a null curve in $\mathbb{E}_{1}^{3}$ with null arc length parameter $s$ and structure function $f(s)$. If the curve $r(s)$ is a slop null helix, the structure function $f(s)$ of $r(s)$ satisfies

$$
\left[\left(\log f_{s}\right)_{s}\right]^{2}-2\left(\log f_{s}\right)_{s s}=2 \kappa(s)=2 a s^{-2}
$$

and can be written as (by an appropriate parameter transformation)

Case $1 f(s)=s^{c}$ or $f(s)=s^{-c}$, for $c \neq 0, \pm 1$ and $2 a=c^{2}-1$;

Case $2 f(s)=\frac{c}{\log s}$ or $f(s)=\frac{\log s}{c}$, for $c \neq 0$ and $2 a=-1$;

Case $3 f(s)=\frac{2}{c} \tan \left(\frac{c}{2} \log s\right)$ or $f(s)=-\frac{2}{c} \tan ^{-1}\left(\frac{c}{2} \log s\right)$, for $c \neq 0$ and $2 a=-c^{2}-1$.

Proof. Solving the equation (35) we can get the conclusion ([10]).

Remark 4.2. In [10], situation like case (3) is inadvertently missed out. 
Proposition 4.1. Let $r(s): \mathbf{I} \rightarrow \mathbb{E}_{1}^{3}$ be a null curve with the null arc length parameter $s$, null curvature function $\kappa(s)$ and null Frenet frame $\{x(s), \alpha(s), y(s)\}$. If there exists some constant vector $0 \neq C_{0} \in \mathbb{E}_{1}^{3}$ such that $\left\langle x(s), C_{0}\right\rangle$ or $\left\langle y(s), C_{0}\right\rangle$ is constant, $\kappa(s)$ is constant and $r(s)$ can be written as the curves given in Theorem 4.1.

Proof. From (8) and $\left\langle x(s), C_{0}\right\rangle=$ constant or $\left\langle y(s), C_{0}\right\rangle=$ constant, we can easily get that $\kappa^{\prime}(s) \equiv 0$.

Theorem 4.5. Let $r(s): \mathbf{I} \rightarrow \mathbb{E}_{1}^{3}$ be a null curve with the null arc length parameter $s$, null curvature function $\kappa(s)$ and null Frenet frame $\{x(s), \alpha(s), y(s)\}$. If the position vector field $r(s)$ of the curve satisfies

$$
r(s)=a_{1}(s) x(s)+a_{3}(s) y(s)
$$

for some functions $a_{1}(s)$ and $a_{3}(s)$, the curve $r(s)$ is called a rectifying null curve. The null curvature function $\kappa(s)$ of the rectifying null curve satisfies

$$
\kappa(s)=a s+b,
$$

where $a \neq 0$ and $b$ are constants. And the curve $r(s)$ can be written as

$$
r(s)=C_{1} \int u^{2}(s) \mathrm{d} s+C_{2} \int u(s) v(s) \mathrm{d} s+C_{3} \int v^{2}(s) \mathrm{d} s,
$$

where $C_{1}, C_{2}, C_{3} \in \mathbb{E}_{1}^{3}$ and when $a>0$

$$
\left\{\begin{array}{l}
u(s)=\sqrt{s} J_{\frac{1}{3}}\left(\frac{\sqrt{2 a}}{3} s^{\frac{3}{2}}\right) \\
v(s)=\sqrt{s} Y_{\frac{1}{3}}\left(\frac{\sqrt{2 a}}{3} s^{\frac{3}{2}}\right)
\end{array}\right.
$$

when $a<0$

$$
\left\{\begin{array}{l}
u(s)=\operatorname{Re}\left(\sqrt{s} Z_{\frac{1}{3}}\left(i \frac{\sqrt{-2 a}}{3} s^{\frac{3}{2}}\right)\right) \\
v(s)=\operatorname{Im}\left(\sqrt{s} Z_{\frac{1}{3}}\left(i \frac{\sqrt{-2 a}}{3} s^{\frac{3}{2}}\right)\right)
\end{array}\right.
$$

where $Z_{\nu}(s)$ is the cylinder function, $J_{\nu}(s)$ is the Bessel function of the first kind, $Y_{\nu}(s)$ is the Bessel function of the second kind.

Proof. From (36) we have

$$
\begin{gathered}
x=a_{1}^{\prime} x+a_{1} \alpha+a_{3}^{\prime} y-a_{3} \kappa \alpha, \\
\left\{\begin{array}{l}
a_{1}^{\prime}=1, \\
a_{1}=a_{3} \kappa, \\
a_{3}^{\prime}=0 .
\end{array}\right.
\end{gathered}
$$

Therefore, the null curvature function $\kappa(s)$ can be written as (37) for some constants $a$ and $b$. From (10), when $\kappa(s)=a s$ (by a parameter transformation we can put $\mathrm{b}=0$ ), the curve $r(s)$ satisfies

$$
\dddot{r}(s)-2 a s \ddot{r}(s)-a \dot{r}(s)=0 .
$$

For the solutions $u(s)$ and $v(s)$ of the Airy-type equation ([15], 2.1.2-1.2, p213)

$$
2 g^{\prime \prime}(s)=\operatorname{asg}(s),
$$

by a direct calculation we know that $u(s) v(s)$ is the solution of the equation

$$
f^{\prime \prime \prime}(s)-2 a s f^{\prime}(s)-a f(s)=0 .
$$

The fundamental solutions of (42) are

$$
g(s)=b_{1} u(s)+b_{2} v(s)=\sqrt{s} Z_{\frac{1}{3}}\left(\frac{\sqrt{2 a}}{3} s^{\frac{3}{2}}\right),
$$


for $a>0$;

$$
g(s)=b_{1} u(s)+b_{2} v(s)=b_{1} \operatorname{Re}\left(\sqrt{s} Z_{\frac{1}{3}}\left(i \frac{\sqrt{-2 a}}{3} s^{\frac{3}{2}}\right)\right)+b_{2} \operatorname{Im}\left(\sqrt{s} Z_{\frac{1}{3}}\left(i \frac{\sqrt{-2 a}}{3} s^{\frac{3}{2}}\right)\right),
$$

for $a<0$; where $b_{1}$ and $b_{2}$ are constants. Where $Z_{\nu}(s)$ is the cylinder function

$$
Z_{\nu}=b_{1} J_{\nu}+b_{2} Y_{\nu}
$$

and $J_{\nu}(s)$ is the Bessel function of the first kind

$$
J_{\nu}(s)=\sum_{k=0}^{\infty} \frac{(-1)^{k}\left(\frac{s}{2}\right)^{\nu+2 k}}{k ! \Gamma(\nu+k+1)}
$$

$Y_{\nu}(s)$ is the Bessel function of the second kind

$$
Y_{\nu}(s)=\frac{J_{\nu}(s) \cos \nu \pi-J_{-\nu}(s)}{\sin \nu \pi} .
$$

Then the solutions of (43) can be written as

$$
f(s)=b_{1} u^{2}(s)+b_{2} u(s) v(s)+b_{3} v^{2}(s),
$$

where $b_{1}, b_{2}$ and $b_{3}$ are constants.

Corollary 4.6. The structure function $f(s)$ of the null curve $r(s)$ in Theorem 4.5 satisfies

$$
\left[\left(\log f_{s}\right)_{s}\right]^{2}-2\left(\log f_{s}\right)_{s s}=2 \kappa(s)=2(a s+b)
$$

and can be written as (by an appropriate parameter transformation)

or

$$
\left\{\begin{array}{l}
f(s)=\int e^{\int \xi(s) \mathrm{d} s} \mathrm{~d} s, \\
\xi(s)=-2 \frac{\mathrm{d}}{\mathrm{d} s}(\ln u(s)),
\end{array}\right.
$$

$$
\left\{\begin{array}{l}
f(s)=\int e^{\int \xi(s) \mathrm{d} s} \mathrm{~d} s, \\
\xi(s)=-2 \frac{\mathrm{d}}{\mathrm{d} s}(\ln v(s)) .
\end{array}\right.
$$

Remark 4.3. The Airy equation is given by

$$
g^{\prime \prime}(\xi)=\xi g(\xi)
$$

Putting

$$
\xi=\left(\frac{a}{2}\right)^{-\frac{3}{2}}\left(\frac{a}{2} s\right)
$$

the equation (42) changes to the Airy equation (53). The solutions of the Airy equation (53) can be written as

$$
g(\xi)=a_{1} \operatorname{Ai}(\xi)+a_{2} \operatorname{Bi}(\xi),
$$

where $\operatorname{Ai}(\xi)$ and $\operatorname{Bi}(\xi)$ are the Airy functions of the first and second kind, respectively. The Airy functions can be represented by

$$
\begin{aligned}
& \operatorname{Ai}(\xi)=\frac{1}{\pi} \int_{0}^{\infty} \cos \left(\frac{1}{3} t^{3}+\xi t\right) \mathrm{d} t \\
& \operatorname{Bi}(\xi)=\frac{1}{\pi} \int_{0}^{\infty}\left[\exp \left(-\frac{1}{3} t^{3}+\xi t\right)+\sin \left(\frac{1}{3} t^{3}+\xi t\right)\right] \mathrm{d} t .
\end{aligned}
$$

(cf. [15], 2.1.2-1.2, p213) 
Theorem 4.7. Let $r(s): \mathbf{I} \rightarrow \mathbb{E}_{1}^{3}$ be a null curve with the null arc length parameter $s$. If the null curvature function $\kappa(s)$ of $r(s)$ satisfies

$$
\kappa(s)=2 a\left(s^{2}+b s+c\right)^{-2},
$$

where $a, b, c$ are constants and $a b c \neq 0$, then the structure function $f(s)$ of $r(s)$ can be written as one of the followings.

1. When the null curvature function $\kappa(s)$ can be written as $2 a\left(s^{2}+p^{2}\right)^{-2}, p^{2}-a>0$, ap $\neq 0$, we have

$$
\left\{\begin{array}{l}
f(s)=\int\left[\exp \left(\int \xi(s) \mathrm{d} s\right)\right] \mathrm{d} s \\
\xi(s)=-2\left(\frac{\mathrm{d} \log \eta(s)}{\mathrm{d} s}\right) \\
\eta(s)=\sqrt{s^{2}+p^{2}} \varphi(\theta) \\
\varphi(\theta)=c_{1} \cos \sqrt{p^{2}-a} \theta+c_{2} \sin \sqrt{p^{2}-a} \theta \\
\theta(s)=\frac{1}{p} \arctan \frac{s}{p}
\end{array}\right.
$$

2. When the null curvature function $\kappa(s)$ can be written as $2 a\left(s^{2}+p^{2}\right)^{-2}, p^{2}-a<0$, ap $\neq 0$, we have

$$
\left\{\begin{array}{l}
f(s)=\int\left[\exp \left(\int \xi(s) \mathrm{d} s\right)\right] \mathrm{d} s \\
\xi(s)=-2\left(\frac{\mathrm{d} \log \eta(s)}{\mathrm{d} s}\right) \\
\eta(s)=\sqrt{s^{2}+p^{2}} \varphi(\theta), \\
\varphi(\theta)=c_{1} \cosh \sqrt{a-p^{2}} \theta+c_{2} \sinh \sqrt{a-p^{2}} \theta \\
\theta(s)=\frac{1}{p} \arctan \frac{s}{p} .
\end{array}\right.
$$

3. When the null curvature function $\kappa(s)$ can be written as $2 a\left(s^{2}+p^{2}\right)^{-2}, p^{2}=a$, ap $\neq 0$, we have

$$
\left\{\begin{array}{l}
f(s)=\int\left[\exp \left(\int \xi(s) \mathrm{d} s\right)\right] \mathrm{d} s \\
\xi(s)=-2\left(\frac{\mathrm{d} \log \eta(s)}{\mathrm{d} s}\right), \\
\eta(s)=\sqrt{s^{2}+p^{2}}\left(c_{1} \frac{1}{p} \arctan \frac{s}{p}+c_{2}\right) .
\end{array}\right.
$$

4. When the null curvature function $\kappa(s)$ can be written as $2 a\left(s^{2}-p^{2}\right)^{-2}, p^{2}+a>$, ap $\neq 0$, we have

$$
\left\{\begin{aligned}
f(s) & =\int\left[\exp \left(\int \xi(s) \mathrm{d} s\right)\right] \mathrm{d} s \\
\xi(s) & =-2\left(\frac{\mathrm{d} \log \eta(s)}{\mathrm{d} s}\right) \\
\eta(s) & =c_{1}|s-p|^{\frac{1+k}{2}}|s+p|^{\frac{1-k}{2}}+c_{2}|s-p|^{\frac{1-k}{2}}|s+p|^{\frac{1+k}{2}} \\
& =\sqrt{\left|s^{2}-p^{2}\right|}\left(c_{1} \cosh \frac{k \theta}{2}+\sinh \frac{k \theta}{2}\right) \\
\theta(s) & =\log \frac{|s-p|}{|s+p|} \\
k^{2} & =\frac{a}{p^{2}}+1
\end{aligned}\right.
$$

5. When the null curvature function $\kappa(s)$ can be written as $2 a\left(s^{2}-p^{2}\right)^{-2}, p^{2}+a<0$, ap $\neq 0$, we have

$$
\left\{\begin{array}{l}
f(s)=\int\left[\exp \left(\int \xi(s) \mathrm{d} s\right)\right] \mathrm{d} s \\
\xi(s)=-2\left(\frac{\mathrm{d} \log \eta(s)}{\mathrm{d} s}\right) \\
\eta(s)=(s+p) \varphi(\theta)=\sqrt{\left|s^{2}-p^{2}\right|}\left(c_{1} \cos \frac{k \theta}{2}+c_{2} \sin \frac{k \theta}{2}\right), \\
\theta(s)=\log \frac{|s-p|}{|s+p|} \\
-k^{2}=\frac{a}{p^{2}}+1
\end{array}\right.
$$


6. When the null curvature function $\kappa(s)$ can be written as $2 a\left(s^{2}-p^{2}\right)^{-2}, p^{2}=-a$, ap $\neq 0$, we have

$$
\left\{\begin{array}{l}
f(s)=\int\left[\exp \left(\int \xi(s) \mathrm{d} s\right)\right] \mathrm{d} s \\
\xi(s)=-2\left(\frac{\mathrm{d} \log \eta(s)}{\mathrm{d} s}\right) \\
\eta(s)=\left(\left|s^{2}-p^{2}\right|\right)^{\frac{1}{2}}\left(c_{1} \frac{1}{p} \log \frac{|s-p|}{|s+p|}+c_{2}\right) .
\end{array}\right.
$$

7. When the null curvature function $\kappa(s)$ can be written as $2 a s^{-1}, a \neq 0$, we have

$$
\left\{\begin{array}{l}
f(s)=\int\left[\exp \left(\int \xi(s) \mathrm{d} s\right)\right] \mathrm{d} s \\
\xi(s)=-2\left(\frac{\mathrm{d} \log \eta(s)}{\mathrm{d} s}\right) \\
\eta(s)=\sqrt{s}\left[c_{1} \operatorname{Re} Z_{1}\left(2 i \sqrt{a} s^{\frac{1}{2}}\right)+c_{2} \operatorname{Im} Z_{1}\left(2 i \sqrt{a} s^{\frac{1}{2}}\right)\right] .
\end{array}\right.
$$

Where $c_{1}, c_{2}$ are constant and $Z_{\nu}(s)$ is the cylinder function.

Remark 4.4. The case 7 in Theorem 4.7 is the same as Theorem 4.5.

Proof. When the null curvature function $\kappa(s)$ of the null curve $r(s)$ is $2 a\left(s^{2}+b s+c\right)^{-2}$, we solve the equation (27) according to the conditions $b^{2}-4 c<0, b^{2}-4 c>0$ and $b^{2}-4 c=0$ as the following.

(a) When $b^{2}-4 c<0$, the null curvature function $\kappa(s)$ of the null curve $x(s)$ can be written as $2 a\left[\left(s+\frac{b}{2}\right)^{2}+p^{2}\right]^{-2}, p^{2}=c-\frac{b^{2}}{4}$. By a parameter transformation $s+s_{0} \rightarrow s$, the equation $(30)$ becomes

$$
\left(s^{2}+p^{2}\right)^{2} \eta^{\prime \prime}-a \eta=0
$$

Putting

$$
\begin{aligned}
\eta(s) & =\sqrt{s^{2}+p^{2}} \varphi(\theta), \\
\theta & =\int \frac{\mathrm{d} s}{s^{2}+p^{2}}=\frac{1}{p} \arctan \frac{s}{p}
\end{aligned}
$$

by a direct calculation we have

$$
\frac{\mathrm{d}^{2} \varphi}{\mathrm{d} \theta^{2}}+\left(p^{2}-a\right) \varphi=0
$$

Case 1. When $p^{2}=c-\frac{b^{2}}{4}>a$, we have $\varphi(\theta)=c_{1} \cos \theta+c_{2} \sin \theta$. Therefore we get the conclusion (58) of Theorem 4.7.

Case 2. When $p^{2}=c-\frac{b^{2}}{4}<a$, we have $\varphi(\theta)=c_{1} \cosh \theta+c_{2} \sinh \theta$. Therefore we get the conclusion (59) of Theorem 4.7.

Case 3. When $p^{2}=c-\frac{b^{2}}{4}=a$, we have $\varphi(\theta)=c_{1} \theta+c_{2}$. Therefore we get the conclusion (60) of Theorem 4.7.

(b) When $b^{2}-4 c>0$, the null curvature function $\kappa(s)$ of the null curve $x(s)$ can be written as $2 a\left[\left(s+\frac{b}{2}\right)^{2}-p^{2}\right]^{-2}, p^{2}=\frac{b^{2}}{4}-c$. By a parameter transformation $s+s_{0} \rightarrow s$, the equation (30) becomes

$$
\left(s^{2}-p^{2}\right)^{2} \eta^{\prime \prime}-a \eta=0 .
$$


Putting

$$
\begin{aligned}
\eta(s) & =(s+p) \varphi(\theta), \\
\theta & =2 p \int \frac{\mathrm{d} s}{s^{2}-p^{2}}=\log \frac{s-p}{s+p}
\end{aligned}
$$

by a direct calculation we have

$$
4 p^{2}\left(\frac{\mathrm{d}^{2} \varphi}{\mathrm{d} \theta^{2}}-\frac{\mathrm{d} \varphi}{\mathrm{d} \theta}\right)-a \varphi=0
$$

Case 4. When $p^{2}=\frac{b^{2}}{4}-c>-a$, we have

$$
\varphi(\theta)=c_{1} \exp \left(\frac{p+\sqrt{p^{2}+a}}{2 p}\right) \theta+c_{2} \exp \left(\frac{p-\sqrt{p^{2}+a}}{2 p}\right) \theta .
$$

Therefore we get the conclusion (61) of Theorem 4.7.

Case 5. When $p^{2}=\frac{b^{2}}{4}-c<-a$, we have

$$
\varphi(\theta)=\left(\exp \frac{1}{2} \theta\right)\left[c_{1} \cos \left(\frac{\sqrt{-p^{2}-a}}{2 p}\right) \theta+c_{2} \sin \left(\frac{\sqrt{-p^{2}-a}}{2 p}\right) \theta\right] .
$$

Therefore we get the conclusion (62) of Theorem 4.7.

Case 6. When $p^{2}=\frac{b^{2}}{4}-c=-a$, the equation (66) becomes

$$
\left(s^{2}-p^{2}\right)^{2} \eta^{\prime \prime}+p^{2} \eta=0 .
$$

When $s^{2}-p^{2}>0$, putting

$$
\begin{aligned}
\eta(s) & =\sqrt{s^{2}-p^{2}} \varphi(\theta), \quad(\text { or } \quad(s+p) \varphi(\theta)) \\
\theta & =2 \int \frac{\mathrm{d} s}{s^{2}-p^{2}}=\frac{1}{p} \log \frac{s-p}{s+p},
\end{aligned}
$$

by a direct calculation we have

$$
\frac{\mathrm{d}^{2} \varphi}{\mathrm{d} \theta^{2}}=0 \quad\left(\text { or } \quad 4\left(\frac{\mathrm{d}^{2} \varphi}{\mathrm{d} \theta^{2}}-\frac{\mathrm{d} \varphi}{\mathrm{d} \theta}\right)+\varphi=0\right) .
$$

Then

$$
\varphi(\theta)=c_{1} \theta+c_{2} \quad\left(\text { or } \quad e^{\frac{1}{2} \theta}\left(c_{1} \theta+c_{2}\right)\right) .
$$

When $s^{2}-p^{2}<0$, putting

$$
\begin{aligned}
\eta(s) & =\sqrt{p^{2}-s^{2}} \varphi(\theta), \quad(\text { or } \quad(s+p) \varphi(\theta)) \\
\theta & =2 \int \frac{\mathrm{d} s}{p^{2}-s^{2}}=\frac{1}{p} \log \frac{p+s}{p-s},
\end{aligned}
$$

by a direct calculation we have

$$
\frac{\mathrm{d}^{2} \varphi}{\mathrm{d} \theta^{2}}=0 \quad\left(\text { or } \quad 4\left(\frac{\mathrm{d}^{2} \varphi}{\mathrm{d} \theta^{2}}+\frac{\mathrm{d} \varphi}{\mathrm{d} \theta}\right)+\varphi=0\right) .
$$

Then

$$
\varphi(\theta)=c_{1} \theta+c_{2} \quad\left(\text { or } \quad e^{-\frac{1}{2} \theta}\left(c_{1} \theta+c_{2}\right)\right) .
$$


Therefore we get the conclusion (63) of Theorem 4.7.

(c) When $b^{2}-4 c=0$, the null curvature function $\kappa(s)$ of the null curve $x(s)$ can be written as $2 a\left(s+\frac{b}{2}\right)^{-1}$. By a parameter transformation $s+s_{0} \rightarrow s$, the equation (30) becomes

$$
s \eta^{\prime \prime}-a \eta=0
$$

Case 7. The solutions of (68) can be given by the cylinder function as the following

$$
\eta(s)=\sqrt{s}\left[c_{1} \operatorname{Re} Z_{1}\left(2 i \sqrt{a} s^{\frac{1}{2}}\right)+c_{2} \operatorname{Im} Z_{1}\left(2 i \sqrt{a} s^{\frac{1}{2}}\right)\right] .
$$

Therefore we get the conclusion (64) of Theorem 4.7.

Theorem 4.8. Let $r(s): \mathbf{I} \rightarrow \mathbb{E}_{1}^{3}$ be a null curve with the null arc length parameter $s$ and the null curvature function $\kappa(s)=2 a\left(s^{2}+b s+c\right)^{-2}$. Then the tangent indicatrix $x(s)=\dot{r}(s)$, as a spacelike curve in $\mathbb{E}_{1}^{3}$, is a rectifying curve.

Proof. For the Frenet frame $\{\alpha(s), \beta(s), \gamma(s)\}$ of a non planar spacelike curve $x(s)$ in Minkowski 3-space, we have the Frenet formulas

$$
\left\{\begin{array}{l}
\dot{x}(s)=\alpha(s), \\
\dot{\alpha}(s)=\tilde{\kappa}(s) \beta(s), \\
\dot{\beta}(s)=-\varepsilon \tilde{\kappa}(s) \alpha(s)+\tilde{\tau}(s) \gamma(s), \\
\dot{\gamma}(s)=\tilde{\tau}(s) \beta(s),
\end{array}\right.
$$

where

$$
\begin{aligned}
\langle\alpha(s), \alpha(s)\rangle & =1, \\
\langle\beta(s), \beta(s)\rangle & =\varepsilon= \pm 1, \\
\langle\gamma(s), \gamma(s)\rangle & =-\varepsilon,
\end{aligned}
$$

and $\tilde{\kappa}(s) \geq 0$. We know that $\tilde{\kappa}(s)$ and $\tilde{\tau}(s)$ are the Frenet curvature function and torsion function of $x(s)$ in Minkowski 3-space $\mathbb{E}_{1}^{3}$. From (8) and (69) we have

$$
\dot{x}=\alpha, \quad \dot{\alpha}=\tilde{\kappa} \beta=\kappa x-y \text {. }
$$

Then

$$
\begin{aligned}
& \tilde{\kappa}=\sqrt{-2 \varepsilon \kappa}, \quad \varepsilon \kappa<0, \\
& \beta=\frac{\kappa x-y}{\sqrt{-2 \varepsilon \kappa}},
\end{aligned}
$$

and

$$
\begin{aligned}
\tilde{\tau} \gamma & =\frac{\kappa^{\prime}}{2 \sqrt{-2 \varepsilon \kappa}} x-\varepsilon \kappa^{\prime}(-2 \varepsilon \kappa)^{-\frac{3}{2}} y \\
& =\frac{\kappa^{\prime}}{2 \sqrt{-2 \varepsilon \kappa}}\left(x+\frac{1}{\kappa} y\right) .
\end{aligned}
$$

If we take

$$
\gamma=\sqrt{\frac{-\varepsilon \kappa}{2}}\left(x+\frac{1}{\kappa} y\right)
$$

we get

$$
\tilde{\tau}=-\frac{\varepsilon}{2}\left(\frac{\kappa^{\prime}}{\kappa}\right) .
$$


Therefore, the Frenet curvature function $\tilde{\kappa}(s)$, torsion function $\tilde{\tau}(s)$ of the tangent indicatrix $x(s)=\dot{r}(s)$ and the null curvature function $\kappa(s)$ of the null curve $r(s)$ in Minkowski 3-space $\mathbb{E}_{1}^{3}$ satisfy

$$
\begin{aligned}
& \tilde{\kappa}(s)=\sqrt{-2 \varepsilon \kappa}, \quad \varepsilon \kappa<0, \\
& \tilde{\tau}(s)=-\frac{\varepsilon}{2}(\log \kappa)^{\prime}=-\frac{\varepsilon}{2}\left(\frac{\kappa^{\prime}}{\kappa}\right)=-\varepsilon\left(\frac{\tilde{\kappa}^{\prime}}{\tilde{\kappa}}\right) .
\end{aligned}
$$

When $\kappa(s)=2 a\left(s^{2}+b s+c\right)^{-2}$, by a direct calculation we have

$$
\frac{\tilde{\tau}(s)}{\tilde{\kappa}(s)}=a_{1} s+a_{2}
$$

where $a_{1} \neq 0$ and $a_{2}$ are constants. The curve $x(s)$ is a rectifying curve $([2],[8])$.

Acknowledgments. The first author is supported by NSFC (No. 11371080), Joint Research of NSFC and NRF; partially supported by Chern Institute of Mathematics and Northeastern University. The second author is supported by the National Research Foundation of Korea (NRF)

Grant NRF-2015R1A2A2A01003491.

\section{References}

1. W. B. Bonnor, Null curves in a Minkowski space-time, Tensor (N. S.), 20(1969), 229-242.

2. B. Y. Chen, When does the position vector of a space curve always lie in its rectifying plane, Amer. Math. Monthly, 110(2003), 147-152.

3. G. Clément, Black holes with a null Killing vector in three-dimensional massive gravity, Classical Quantum Gravity, 26(2009), 165002(11pp).

4. A. Ferrández, A. Giménez, P. Lucas, Null generalized helices in Lorentz-Minkowski spaces, J. Phys. A: Math. Gen., 35(2002), no. 39, 8243-8251.

5. A. Ferrández, A. Giménez, P. Lucas, Geometrical particle models on 3D null curves Phys. Lett. B, 543(2002), 311-317.

6. A. Ferrández, A. Giménez, P. Lucas, Relativistic particles with rigidity along light-like curves, In HORIZONS IN WORLD PHYSICS, Vol. 245, Ch. 6, Tori V. Lynch (Editor), Nova Science Publishers Inc., 2004, ISBN:159454-063-2.

7. A. Ferrández, A. Giménez, P. Lucas, Relativistic particles and the geometry of 4d null curves, J. Geom. Phys., $\mathbf{5 7}(10)(2007), 2124-2135$.

8. Jun-ichi Inoguchi, Sungwook Lee, Null curves in Monkowski 3-space, International Electronoc Journal of Geometry, 2(2008), 40-83.

9. Huili Liu, Curves in the lightlike cone, Contrib. Algebr. Geom., 45(2004), 291-303.

10. Huili Liu, Qingxian Meng, Representation formulas of curves in a 2 and 3 dimensional lightlike cone, Results Math., 59(2011), 437-451.

11. Armen Nersessian, Large massive 4 d particle with torsion and conformal mechanics, Physics Letters B, 473, Issues 1-2, 27 January 2000, Pages 94-101.

12. Armen Nersessian, R. Manvelyan, H.J.W. Müller-Kirsten, Particle with torsion on $3 d$ null-curves, Nuclear Physics B - Proceedings Supplements, Volume 88, Issues 11́C3, June 2000, Pages 381-384.

13. Armen Nersessian, Eduardo Ramos, Massive spinning particles and the geometry of null curves, Phys. Lett. B, 445(1998), 123-128.

14. Armen Nersessian, Eduardo Ramos, A geometrical particle model for anyons, Modern Phys. Lett. A, 14 (1999), No. 29, 2033-2037.

15. A. D. Polyanin, V. F. Zaitsev Handbook of Exact Solutions for Ordinary Differential Equations, second edition, 2003, Chapman \& Hall/CRC. 